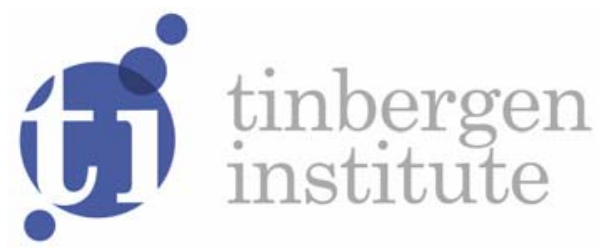

\title{
The Reverse TAL-family of Rules for Bankruptcy Problems
}

René van den Brink'

Juan D. Moreno-Ternero²

' Faculty of Economics and Business Administration, VU University Amsterdam, and Tinbergen Institute, The Netherlands;

2 Universidad Pablo de Olavide, Spain, and Université Catholique de Louvain, Belgium. 
Tinbergen Institute is the graduate school and research institute in economics of Erasmus University Rotterdam, the University of Amsterdam and VU University Amsterdam.

More TI discussion papers can be downloaded at http://www.tinbergen.nl

Tinbergen Institute has two locations:

Tinbergen Institute Amsterdam

Gustav Mahlerplein 117

1082 MS Amsterdam

The Netherlands

Tel.: +31(0)20525 1600

Tinbergen Institute Rotterdam

Burg. Oudlaan 50

3062 PA Rotterdam

The Netherlands

Tel.: +31(0)10 4088900

Fax: +31(0)10 4089031 


\title{
The reverse TAL-family of rules for bankruptcy problems*
}

\author{
Rene van den Brink ${ }^{\dagger} \quad$ Juan D. Moreno-Ternero ${ }^{\ddagger}$
}

December 7, 2016

\begin{abstract}
This paper analyzes a family of rules for bankruptcy problems that generalizes the socalled reverse Talmud rule and encompasses both the constrained equal-awards rule and the constrained equal-losses rule. The family, introduced by van den Brink et al., [Characterization of the reverse Talmud bankruptcy rule by exemption and exclusion properties, European Journal of Operational Research 228 (2013), 413-417], is a counterpart to the so-called TAL-family of rules, introduced and studied by Moreno-Ternero and Villar [The TAL-family of rules for bankruptcy problems, Social Choice and Welfare 27 (2006) 231249], and it is included within the so-called CIC-family of rules introduced by Thomson [Two families of rules for the adjudication of conflicting claims, Social Choice and Welfare 31 (2008) 667-692]. We provide a systematic study of the structural properties of the rules within the family, as well as its connections with the existing related literature.
\end{abstract}

JEL numbers: D63.

Keywords: Bankruptcy problems, reverse TAL-family, reverse Talmud rule, Equal awards, Equal losses.

*Moreno-Ternero acknowledges financial support from the Spanish Ministry of Economy and Competitiveness (ECO2014-57413-P).

${ }^{\dagger}$ Department of Econometrics and Tinbergen Institute, VU University Amsterdam.

†Department of Economics, Universidad Pablo de Olavide and CORE, Université catholique de Louvain. 


\section{Introduction}

The problem of adjudicating conflicting claims refers to a situation in which one has to distribute a good whose available amount is not enough to cover all agents' demands on it. This is a classic allocation problem, which encompasses many different situations, like the bankruptcy of a firm (our running interpretation throughout this paper; hence its title), or the collection of a given amount of taxes. The reader is referred to Thomson $(2003,2014,2015 \mathrm{a})$ for extensive reviews and surveys of the sizable literature dealing with this problem, which originated in O'Neill (1982).

A classical rule to solve problems of adjudicating conflicting claims is the so-called Talmud rule (e.g., Aumann and Maschler, 1985), which applies equal division until the claimant with the smallest claim has obtained one half of her claim. Then, that agent stops receiving additional units and the remaining amount is divided equally among the other agents until the claimant with the second smallest claim gets one half of her claim. The process continues until every agent has received one half of her claim, or the available amount is distributed. If there is still something left after this process, agents are invited back to receive additional shares. Now agents receive additional amounts sequentially starting with those with larger claims and applying equal division of their losses.

One natural way of generalizing the Talmud rule would be obtained by moving the threshold in the above definition from one half to any other possible fraction (of the aggregate and individual incomes). In doing so, we would obtain a non-countable set of piece-wise linear rules ranging from the so-called constrained equal-awards rule to the so-called constrained equallosses rule (and having the Talmud rule in the middle). The resulting family of rules, known as the TAL-family, was introduced by Moreno-Ternero and Villar (2006a), who provided a systematic study of the structural properties of the rules within the family. ${ }^{1}$ Thomson (2008) analyzes a more general family encompassing this one, known as the ICI-family, which impose that the evolution of each claimant's award, as a function of the amount to divide, is increasing first, constant next and finally increasing again. ${ }^{2}$ Moreno-Ternero (2011a) considers another generalization of the TAL-family in which the slope of the pieces might be different.

\footnotetext{
${ }^{1}$ See also Moreno-Ternero and Villar (2006b) and Moreno-Ternero (2007, 2011b).

${ }^{2}$ More recently, Huijink et al., (2015) have given an alternative definition of the rules in such a family, as claim-and-right rules, which give a specific interpretation to the concept of baselines formalized earlier by Hougaard et al., (2012, 2013a, 2013b). See also Pulido et al., (2002, 2008), Bergantiños and Lorenzo (2008) and Timoner and Izquierdo (2016) for related notions.
} 
The Talmud rule has a natural counterpart rule in which the equal awards and equal losses principles are applied in the reverse order. More precisely, the so-called reverse Talmud rule (e.g., Chun et al., 2001) originates when, for each claims vector, we apply the equal losses principle in the lower half of the range of the endowment, and the equal awards principle to the upper half. As for the Talmud rule, half-claims are used instead of the claims themselves. Therefore, the same natural idea considered above to generalize the Talmud rule could be considered to generalize the reverse Talmud rule, as recently suggested by van den Brink et al., (2013). That process gives rise to a new family of rules, the reverse TAL-family, that will be the object of this study. Such a family also comprises a non-countable set of piece-wise linear rules, ranging from the constrained equal-awards rule to the constrained equal-losses rule, but this time having the reverse Talmud rule in the middle. The family is included within a more general family, known as the CIC-family also introduced by Thomson (2008). All CIC-rules impose that the evolution of each claimant's award, as a function of the amount to divide, is constant first, increasing next and finally constant again.

The aim of this paper is to present a systematic study of the structural properties of the rules within the reverse TAL-family, as well as its connections with the existing related literature. In particular, we shall show the similarities and differences with respect to its counterpart TAL-family of rules. As we shall see, some of the results we obtain will be derived from the techniques developed in Moreno-Ternero and Villar (2006a,b) and Thomson (2008).

The paper is organized as follows. Section 2 describes the reference model and presents the reverse TAL-family. Section 3 analyzes the structural properties of the rules in this family. Section 4 relates the family to other existing families in the literature. Section 5 concludes. All the proofs are relegated to an appendix.

\section{The model}

We study bankruptcy problems in a variable-population model. The set of potential claimants, or agents, is identified with the set of natural numbers $\mathbb{N}$. Let $\mathcal{N}$ be the class of finite subsets of $\mathbb{N}$, with generic element $N$. Let $n$ denote the cardinality of $N$. For each $i \in N$, let $c_{i} \in \mathbb{R}_{+}$be $i$ 's claim and $c \equiv\left(c_{i}\right)_{i \in N}$ the claims profile. ${ }^{3}$ A (bankruptcy) problem is a triple consisting of a population $N \in \mathcal{N}$, a claims profile $c \in \mathbb{R}_{+}^{n}$, and an endowment $E \in \mathbb{R}_{+}$such that $\sum_{i \in N} c_{i} \geq E$. Let $C \equiv \sum_{i \in N} c_{i}$. To avoid unnecessary complication, we assume $C>0$. Let $\mathcal{D}^{N}$ be the domain

\footnotetext{
${ }^{3}$ For each $N \in \mathcal{N}$, each $M \subseteq N$, and each $z \in \mathbb{R}^{n}$, let $z_{M} \equiv\left(z_{i}\right)_{i \in M}$. For each $i \in N$, let $z_{-i} \equiv z_{N \backslash\{i\}}$.
} 
of bankruptcy problems with population $N$ and $\mathcal{D} \equiv \bigcup_{N \in \mathcal{N}} \mathcal{D}^{N}$.

Given a problem $(N, c, E) \in \mathcal{D}^{N}$, an allocation is a vector $x \in \mathbb{R}^{n}$ satisfying the following two conditions: (i) for each $i \in N, 0 \leq x_{i} \leq c_{i}$ and (ii) $\sum_{i \in N} x_{i}=E$. We refer to (i) as boundedness and (ii) as balance. A rule on $\mathcal{D}, R: \mathcal{D} \rightarrow \bigcup_{N \in \mathcal{N}} \mathbb{R}^{n}$, associates with each problem $(N, c, E) \in \mathcal{D}$ an allocation $R(N, c, E)$ for the problem. Each rule $R$ has a dual rule $R^{*}$ defined as $R^{*}(N, c, E)=c-R(N, c, C-E)$, for each $(N, c, E) \in \mathcal{D}$.

We now consider some classical rules. The constrained equal-awards rule distributes the amount equally among all agents, subject to no agent receiving more than she claims. The constrained equal-losses rule imposes that losses are as equal as possible subject to no one receiving a negative amount. Finally, the Talmud rule behaves like the former or the latter rule, depending on whether the amount to divide falls short or exceeds one half of the aggregate claim, using half-claims instead of claims. Formally,

The constrained equal-awards rule, $A$, selects for each $(N, c, E) \in \mathcal{D}$, the vector $\left(\min \left\{c_{i}, \lambda\right\}\right)_{i \in N}$, where $\lambda>0$ is chosen so that $\sum_{i \in N} \min \left\{c_{i}, \lambda\right\}=E$.

The constrained equal-losses rule, $L$, selects for each $(N, c, E) \in \mathcal{D}$, the vector $\left(\max \left\{0, c_{i}-\right.\right.$ $\lambda\})_{i \in N}$, where $\lambda>0$ is chosen so that $\sum_{i \in N} \max \left\{0, c_{i}-\lambda\right\}=E$.

The Talmud rule, $T$, selects for each $(N, c, E) \in \mathcal{D}$, the vector $\left(\min \left\{\frac{1}{2} c_{i}, \lambda\right\}\right)_{i \in N}$ if $E \leq$ $\frac{1}{2} C$ and the vector $\left(\max \left\{\frac{1}{2} c_{i}, c_{i}-\mu\right\}\right)_{i \in N}$ if $E \geq \frac{1}{2} C$, where $\lambda$ and $\mu$ are chosen so that $\sum_{i \in N} T_{i}(N, c, E)=E$.

The Talmud rule can also be given the following representation, which will be useful for the ensuing discussion. For each $(N, c, E) \in \mathcal{D}$,

$$
T(N, c, E)= \begin{cases}A\left(N, \frac{1}{2} c, E\right) & \text { if } E \leq \frac{1}{2} C \\ \frac{1}{2} c+L\left(N, \frac{1}{2} c, E-\frac{1}{2} C\right) & \text { if } E \geq \frac{1}{2} C\end{cases}
$$

The TAL-family generalizes the idea underlying the Talmud rule by applying the same principle to $E$ and $\theta C$, and using $\theta c$ as the switch point, for each value of $\theta$ within the interval $[0,1]$. Formally:

The $\boldsymbol{T A L}$-family consists of all rules with the following form: There exists $\theta \in[0,1]$ such that, for each $(N, c, E) \in \mathcal{D}$, and each $i \in N$,

$$
T_{i}^{\theta}(N, c, E)= \begin{cases}\min \left\{\theta c_{i}, \lambda\right\} & \text { if } E \leq \theta C \\ \max \left\{\theta c_{i}, c_{i}-\mu\right\} & \text { if } E \geq \theta C\end{cases}
$$


where $\lambda$ and $\mu$ are chosen so that $\sum_{i \in N} T_{i}^{\theta}(N, c, E)=E$.

Alternatively, the TAL-family can be expressed as follows. For each $(N, c, E) \in \mathcal{D}$,

$$
T^{\theta}(N, c, E)= \begin{cases}A(N, \theta c, E) & \text { if } E \leq \theta C \\ \theta c+L(N,(1-\theta) c, E-\theta C) & \text { if } E \geq \theta C\end{cases}
$$

Note that the constrained equal-awards rule corresponds to the case $\theta=1\left(T^{1}=A\right)$, whereas the constrained equal-losses rule corresponds to the case $\theta=0\left(T^{0}=L\right)$. Obviously, the Talmud rule is obtained for $\theta=\frac{1}{2}\left(T^{\frac{1}{2}}=T\right)$.

The reverse Talmud rule implements a sort of reverse protocol to the one provided by the Talmud rule, switching the roles between the equal awards and equal losses principles. Formally, The Reverse Talmud rule, $R T$, selects for each $(N, c, E) \in \mathcal{D}$, the vector

$$
R T(N, c, E)= \begin{cases}L\left(N, \frac{1}{2} c, E\right) & \text { if } E \leq \frac{1}{2} C \\ \frac{1}{2} c+A\left(N, \frac{1}{2} c, E-\frac{1}{2} C\right) & \text { if } E \geq \frac{1}{2} C\end{cases}
$$

We conclude this inventory of rules by presenting a family of rules that generalizes the reverse Talmud rule (and encompasses the constrained equal-awards rule and the constrained equal-losses rule) in the same way as the TAL-family presented above generalized the Talmud rule. Formally:

The reverse $\mathbf{T A L}$-family consists of all rules with the following form: There exists $\theta \in[0,1]$ such that, for each $(N, c, E) \in \mathcal{D}$, and each $i \in N$,

$$
R T_{i}^{\theta}(N, c, E)= \begin{cases}\max \left\{\theta c_{i}-\lambda, 0\right\} & \text { if } E \leq \theta C \\ \theta c_{i}+\min \left\{(1-\theta) c_{i}, \mu\right\} & \text { if } E \geq \theta C\end{cases}
$$

where $\lambda$ and $\mu$ are chosen so that $\sum_{i \in N} R T_{i}^{\theta}(N, c, E)=E$.

Alternatively, the reverse TAL-family can be expressed as follows. For each $(N, c, E) \in \mathcal{D}$,

$$
R T^{\theta}(N, c, E)=\left\{\begin{array}{ll}
L(N, \theta c, E) & \text { if } E \leq \theta C \\
\theta c+A(N,(1-\theta) c, E-\theta C) & \text { if } E \geq \theta C
\end{array},\right.
$$

or, equivalently,

$$
R T^{\theta}(N, c, E)=L(N, \theta c, \min \{E, \theta C\})+A(N,(1-\theta) c, \max \{E-\theta C, 0\}) .
$$


It is straightforward to see that all rules within the family are well defined. Note that the constrained equal-awards rule corresponds to the case $\theta=0\left(R T^{0}=A\right)$, whereas the constrained equal-losses rule corresponds to the other extreme value, $\theta=1\left(R T^{1}=L\right)$. Obviously, the Reverse Talmud rule is obtained for $\theta=\frac{1}{2}\left(R T^{1 / 2}=R T\right)$.

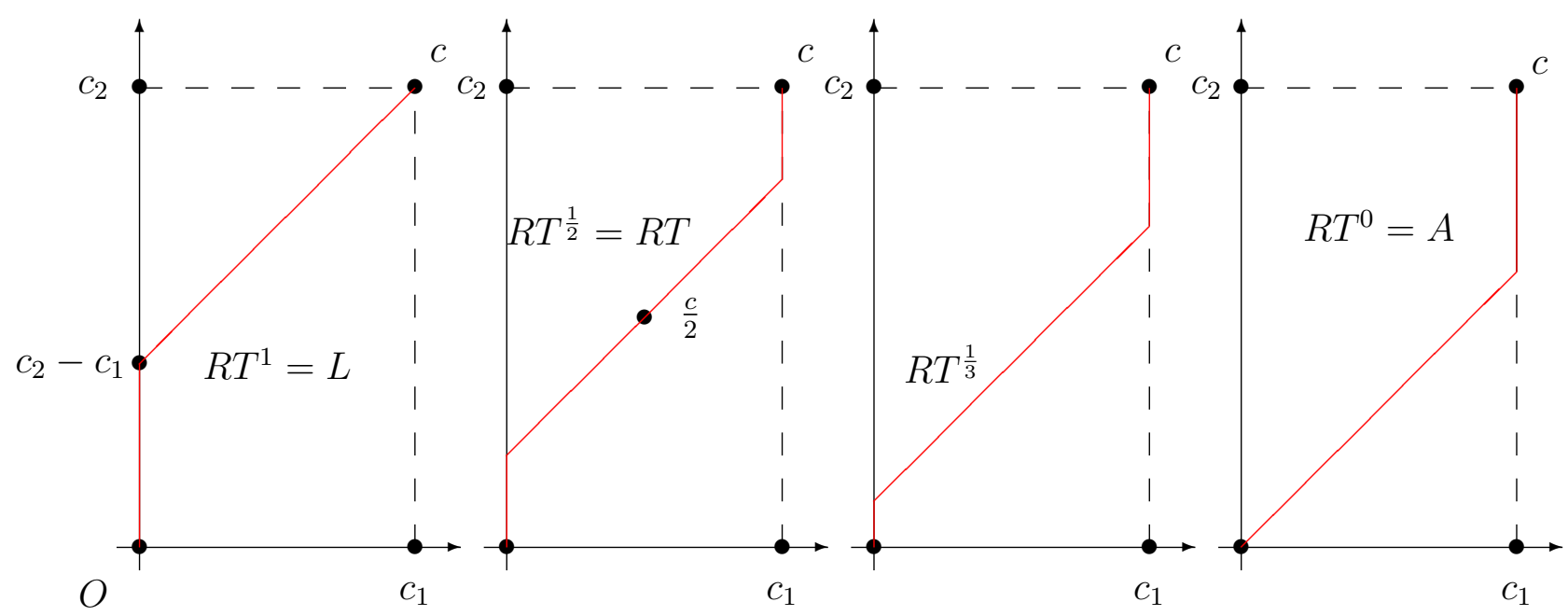

Figure 1: Rules in the two-claimant case. This figure illustrates the "path of awards" of some rules within the reverse TAL-family for $N=\{1,2\}$ and $c \in \mathbb{R}_{+}^{n}$ with $c_{1}<c_{2}$. The path of awards for $c$ (the locus of the awards vector chosen by a rule as the amount to divide $E$ varies from 0 to $c_{1}+c_{2}$ ) of $R T^{1}=L$ follows the vertical axis until the average loss coincides with the lowest claim, i.e., until $E=c_{2}-c_{1}$. After that, it follows the line of slope 1 until it reaches the vector of claims. The path of awards of $R T^{1 / 2}=R T$ follows the vertical line until claimant 2 obtains $\frac{c_{2}-c_{1}}{2}$. Then, it follows the line of slope 1 (crossing $c / 2$ ) until claimant 1 is fully awarded, from where it follows vertically until it reaches the vector of claims. The path of awards of $R T^{1 / 3}$ follows the vertical line until claimant 2 obtains $\frac{c_{2}-c_{1}}{3}$. Then, it follows the line of slope 1 until claimant 1 is fully awarded, from where it follows vertically until it reaches the vector of claims. Finally, the path of awards of $R T^{0}=A$ follows the $45^{\circ}$ line until it gives the whole claim to the lowest claimant, i.e. until $E=2 c_{1}$, from where it is vertical until it reaches the vector of claims.

\section{Properties of the reverse TAL-family}

We devote this section to analyze the structural properties of the rules within the reverse TAL-family. 


\subsection{Duality relationship and parametric representation}

We first convey a precise duality relationship between the members of the reverse TAL-family. ${ }^{4}$

Proposition 1. For each $\theta \in[0,1]$, it follows that the dual rule of $R T^{\theta}$ is $R T^{1-\theta}$.

Two well-known results are immediately derived from this duality relationship. One is that $L$ and $A$ are dual rules. Another is that $R T$ is self-dual (in fact, there is no other self-dual rule in the reverse TAL-family).

As defined by Young (1987), a rule is parametric if the $i$ th agent's award is a function that only depends on $c_{i}$ and a parameter $\lambda$, which is related to the size of the amount to divide. More precisely:

$A$ rule $R$ is parametric if there exists a function $f:[a, b] \times \mathbb{R}_{+} \rightarrow \mathbb{R}_{+}$, where $[a, b] \subset \mathbb{R} \cup\{ \pm \infty\}$, continuous and weakly monotonic in its first argument, such that:

(i) $R_{i}(N, c, E)=f\left(\lambda, c_{i}\right)$ for each $(N, c, E) \in \mathcal{D}$ and for some $\lambda \in[a, b]$;

(ii) $f(a, x)=0$, for each $x \in \mathbb{R}_{+}$; and

(iii) $f(b, x)=x$, for each $x \in \mathbb{R}_{+}$.

Analogously to what happens with the TAL-family (see Moreno-Ternero and Villar, 2006a), all rules within the reverse TAL-family are parametric. To check this, let $\theta \in[0,1]$ be given and define $f^{\theta}: \mathbb{R} \cup\{ \pm \infty\} \times \mathbb{R}_{+} \rightarrow \mathbb{R}_{+}$as follows:

$$
f^{\theta}\left(\lambda, c_{i}\right)=\left\{\begin{array}{ll}
\max \left\{0, \lambda+\theta c_{i}\right\} & \text { if } \lambda<0 \\
\min \left\{c_{i}, \lambda+\theta c_{i}\right\} & \text { if } \lambda \geq 0
\end{array} .\right.
$$

Clearly, $f^{\theta}$ is continuous and weakly monotonic in its first argument, with $\lim _{\lambda \rightarrow-\infty} f^{\theta}\left(\lambda, c_{i}\right)=$ 0 and $\lim _{\lambda \rightarrow+\infty} f^{\theta}\left(\lambda, c_{i}\right)=c_{i}$ for each $c_{i} \in \mathbb{R}_{+}$. As a result, the Darboux property shows that, for each $(N, c, E) \in \mathcal{D}$, there exists $\lambda_{0} \in \mathbb{R}_{+}$such that $E=\sum_{i \in N} f^{\theta}\left(\lambda_{0}, c_{i}\right)$. Thus, $f^{\theta}\left(\lambda_{0}, c_{i}\right)=R T_{i}^{\theta}(N, c, E)$, which shows that $f^{\theta}$ is a parametric representation of $R T^{\theta}$.

\subsection{Basic properties}

Young (1987) characterized the parametric rules as those satisfying the following three properties: equal treatment of equals (agents with equal claims should receive equal amounts), continuity (small changes in the parameters of the problem should not induce large changes

\footnotetext{
${ }^{4}$ This duality relationship, which was already mentioned by van den Brink et al., (2013), is similar to the one exhibited for the TAL-family (see Proposition 1 at Moreno-Ternero and Villar, 2006a).
} 
in the corresponding allocation) and consistency (if some claimants leave with their awards and the problem of dividing among the remaining claimants what is left is considered, these claimants should receive the same awards as initially).

Formally, a rule $R$ satisfies equal treatment of equals if for each $(N, c, E) \in \mathcal{D}$, and each pair $i, j \in N$, we have $R_{i}(N, c, E)=R_{j}(N, c, E)$, whenever $c_{i}=c_{j}$. A rule $R$ satisfies continuity if, for each sequence $\left\{\left(N, c^{k}, E^{k}\right)\right\}$ of problems in $\mathcal{D}$, and each $(N, c, E) \in \mathcal{D}$, if $\left(N, c^{k}, E^{k}\right) \rightarrow(N, c, E)$, then $R\left(N, c^{k}, E^{k}\right) \rightarrow R(N, c, E)$. A rule $R$ is consistent if for each $(N, c, E) \in \mathcal{D}$, each $M \subset N$, and each $i \in M$, we have $R_{i}(N, c, E)=R_{i}\left(M, c_{M}, E_{M}\right)$, where $E_{M}=\sum_{i \in M} R_{i}(N, c, E)$.

Therefore, the above allows us to show that all rules within the reverse TAL-family satisfy equal treatment of equals, continuity, and consistency. As a matter of fact, the rules also satisfy two strengthenings of equal treatment of equals; namely, anonymity (any "renaming" of claimants should be accompanied by a parallel reassignment of awards) and order preservation (agents with larger claims receive larger awards but face larger losses too). They also satisfy the basic property of scale invariance (if claims and endowment are multiplied by the same positive number, then so should all awards).

Formally, a rule $R$ is anonymous if for each $(N, c, E) \in \mathcal{D}$, each permutation $\pi \in \Pi^{N}$, and $i \in N, R_{\pi(i)}\left(N,\left(c_{\pi(i)}\right)_{i \in N}, E\right)=R_{i}(N, c, E)$. A rule $R$ is order preserving, if for each $(N, c, E) \in \mathcal{D}$ and each pair $i, j \in N, c_{i} \geq c_{j}$ implies that $R_{i}(N, c, E) \geq R_{j}(N, c, E)$ and $c_{i}-R_{i}(N, c, E) \geq c_{j}-R_{j}(N, c, E)$. A rule is scale invariant, if for each $(N, c, E) \in \mathcal{D}$ and $\lambda \in \mathbb{R}_{+}, R(N, \lambda c, \lambda E)=\lambda R(N, c, E)$.

Proposition 2. All rules within the reverse TAL-family satisfy anonymity, order preservation, continuity, scale invariance, and consistency.

\subsection{Monotonicity properties}

We now consider a set of monotonicity properties that are also satisfied by all members of the family. They are the following.

Resource monotonicity: if there is more to be divided, nobody should lose; Claims monotonicity: if an agent's claim increases, she should receive at least as much as she did initially; Linked claim-resource monotonicity: if an agent's claim and the endowment increase by the same amount, while the claims of the other agents stay the same, the agent's award should increase by at most that amount; Population monotonicity: if new claimants arrive, each claimant 
initially present should receive at most as much as he did initially; Linked resource-population monotonicity: if new claimants arrive and the endowment increases by the sum of their claims, then each claimant initially present should receive at least as much as he did initially; Resourceand-population uniformity: the arrival of new agents should affect all the incumbent agents in the same direction;

Formally, a rule $R$ is resource monotonic if, for each $(N, c, E) \in \mathcal{D}$ and each $E^{\prime}>E$, with $E^{\prime} \leq C$, we have $R(N, c, E) \leq R\left(N, c, E^{\prime}\right)$. A rule $R$ is claims monotonic if, for each $(N, c, E) \in$ $\mathcal{D}$, each $i \in N$, and each $c_{i}^{\prime}>c_{i}$, we have $R_{i}\left(N,\left(c_{i}^{\prime}, c_{N \backslash\{i\}}\right), E\right) \geq R_{i}\left(N,\left(c_{i}, c_{N \backslash\{i\}}\right), E\right)$. A rule $R$ satisfies linked claims-resource monotonicity if, for each $(N, c, E) \in \mathcal{D}$ and each $i \in N$, $R_{i}\left(N,\left(c_{i}+\varepsilon, c_{N \backslash\{i\}}\right), E+\varepsilon\right) \leq R_{i}(N, c, E)+\varepsilon$. A rule $R$ is population monotonic if, for each $(N, c, E) \in \mathcal{D}$ and each $\left(N^{\prime}, c^{\prime}, E\right) \in \mathcal{D}$ such that $N \subseteq N^{\prime}$ and $c_{N}^{\prime}=c$, then $R_{i}\left(N^{\prime}, c^{\prime}, E\right) \leq$ $R_{i}(N, c, E)$, for each $i \in N$. A rule $R$ satisfies linked resource-population monotonicity if for each $(N, c, E) \in \mathcal{D}$ and each $\left(N^{\prime}, c^{\prime}, E^{\prime}\right) \in \mathcal{D}$ such that $N \subseteq N^{\prime}, c_{N}^{\prime}=c$, and $E=E^{\prime}$, then $R_{i}(N, c, E) \leq R_{i}\left(N^{\prime}, c^{\prime}, E+\sum_{N^{\prime} \backslash N} c_{j}^{\prime}\right)$, for each $i \in N$. Finally, a rule $R$ satisfies resource-and-population uniformity if for each $(N, c, E) \in \mathcal{D}$ and each $\left(N^{\prime}, c^{\prime}, E^{\prime}\right) \in \mathcal{D}$ such that $N \subseteq N^{\prime}$ and $c_{N}^{\prime}=c$, then, either $R_{i}\left(N^{\prime}, c^{\prime}, E^{\prime}\right) \leq R_{i}(N, c, E)$, for each $i \in N$, or $R_{i}\left(N^{\prime}, c^{\prime}, E^{\prime}\right) \geq R_{i}(N, c, E)$, for each $i \in N$.

Similar properties turned out to be very strong in other domains of problems, sometimes even being incompatible with very elementary requirements of efficiency and fairness (e.g., Thomson, 2013). It turns out, however, that all rules within the reverse TAL-family satisfy these properties, which are reasonably weak in the context of bankruptcy problems.

Proposition 3. All rules within the reverse TAL-family satisfy resource monotonicity, claims monotonicity, linked claim-resource monotonicity, population monotonicity, linked resourcepopulation monotonicity, and resource-and-population uniformity

\subsection{Independence properties}

We now consider two dual independence properties, known as minimal rights first and claims truncation invariance. ${ }^{5}$ The former postulates that the part of a claim that is above the amount to divide should be ignored. That is, $R(N, c, E)=R(N, t(N, c, E), E)$, where $t_{i}(N, c, E)=$ $\min \left\{E, c_{i}\right\}$ for each $i \in N$. The latter ensures each agent the portion of the endowment that

\footnotetext{
${ }^{5}$ We say that a property $P^{*}$ is the dual of property $P$ if, for each rule $R$, it holds that $R$ satisfies $P$ if and only if $R^{*}$ satisfies $P^{*}$. A property is self-dual if it coincides with its dual.
} 
is left to her when the claims of all other agents are fully honored (provided this amount is nonnegative) and divides the remainder according to revised claims. Formally,

$$
R(N, c, E)=m(N, c, E)+R(N, c-m(N, c, E), E-M(N, c, E)),
$$

where $m_{i}(N, c, E)=\max \left\{0, E-\sum_{j \in N \backslash\{i\}} c_{j}\right\}$, for each $i \in N, m(N, c, E)=\left[m_{i}(N, c, E)\right]_{i \in N}$ and $M(N, c, E)=\sum_{i \in N} m_{i}(N, c, E)$.

As the following result shows, these two properties are highly disruptive within the reverse TAL-family.

Proposition 4. The following statements hold:

(i) The only rule in the reverse TAL-family that satisfies claims truncation invariance is $R T^{0}=A$.

(ii) The only rule in the reverse TAL-family that satisfies minimal rights first is $R T^{1}=L$.

Proposition 4 shows an interesting difference between the reverse TAL-family and the TALfamily. Whereas there is only one rule in the reverse TAL-family that satisfies claims truncation invariance (the constrained equal-awards rule), all rules in the TAL-family with parameter $\theta \in\left[\frac{1}{2}, 1\right]$ satisfy claims truncation invariance (Moreno-Ternero and Villar, 2006a; Theorem 1). Similarly, there is only one rule in the reverse TAL-family that satisfies minimal rights first (the constrained equal-losses rule), while all rules in the TAL-family with parameter $\theta \in\left[0, \frac{1}{2}\right]$ satisfy this axiom. Thus, within the TAL-family every rule satisfies at least one of these two axioms (with the Talmud rule being the only one that satisfies both), while in the reverse TAL-family, besides the constrained equal-awards and constrained equal-losses rule, none of the rules satisfy any of these two axioms.

\subsection{Lower and upper bounds}

Although lower bounds have long been used within the theory of fair allocation, they have only been recently explored for bankruptcy problems. A focal lower bound is the so-called average truncated lower bound on awards, which is somewhat related to the claims truncation invariance axiom considered above. It ensures each agent a minimal share of her individual claim, no matter what the other claims are. In particular, for a problem involving $n$ agents, it establishes that any agent holding a feasible claim (a claim not larger than the amount to divide) will get at least one $n$th of her claim. And also that those agents whose individual claims 
are unfeasible will get at least one $n$th of the amount to divide. ${ }^{6}$ Formally, a rule $R$ satisfies average truncated lower bound on awards if, for each $(N, c, E) \in \mathcal{D}, R_{i}(N, c, E) \geq \frac{1}{n} \min \left\{c_{i}, E\right\}$. Its dual property is also an interesting one. This property provides an upper bound to each claimant involved in the problem. Formally, a rule $R$ satisfies average truncated lower bound on losses if, for each $(N, c, E) \in \mathcal{D}, R_{i}(N, c, E) \leq c_{i}-\frac{1}{n} \min \left\{c_{i}, C-E\right\}$. The next result describes the behavior of the family with respect to these properties.

Proposition 5. The following statements hold:

(i) The only rule in the reverse TAL-family that satisfies average truncated lower bound on awards is $R T^{0}=A$.

(ii) The only rule in the reverse TAL-family that satisfies average truncated lower bound on losses is $R T^{1}=L$.

Proposition 5 also shows an interesting difference between the reverse TAL-family and the TAL-family. Whereas there is only one rule in the reverse TAL-family that satisfies average truncated lower bound on awards (the constrained equal-awards rule), all rules in the TALfamily with parameter $\theta \in\left[\frac{1}{2}, 1\right]$ satisfy average truncated lower bound on awards (MorenoTernero and Villar, 2006a; Theorem 2). Similarly, there is only one rule in the reverse TALfamily that satisfies average truncated lower bound on losses (the constrained equal-losses rule), while all rules in the TAL-family with parameter $\theta \in\left[0, \frac{1}{2}\right]$ satisfy this axiom. Thus, within the TAL-family, every rule satisfies at least one of these two axioms (with the Talmud rule being the only one that satisfies both), while in the reverse TAL-family, besides the constrained equal-awards and constrained equal-losses rule, none of the rules satisfy any of these two axioms.

\subsection{Composition properties}

We now consider two additional properties dealing with the solvability of a bankruptcy problem in stages. To motivate these properties think of the following situation: after having divided the allocation of the available amount among its creditors, it turns out that the actual value of the amount is larger than was initially assumed. Then, two options are open: either the tentative division is cancelled altogether and the actual problem is solved, or we add to the initial distribution the result of applying the rule to the remaining amount. The requirement formulated next is that both ways of proceeding should result in the same awards vectors.

\footnotetext{
${ }^{6}$ The property was introduced by Moreno-Ternero and Villar (2004) under the name of securement.
} 
Formally, a rule $R$ satisfies composition up if, for each $(N, c, E) \in \mathcal{D}$, and each pair $E_{1}, E_{2} \in \mathbb{R}_{++}$ such that $E_{1}+E_{2}=E, R(N, c, E)=R\left(N, c, E_{1}\right)+R\left[N, c-R\left(N, E_{1}, c\right), E_{2}\right]$.

Think now of the dual case. Namely, after having divided the available amount among its creditors one finds that the actual value of the amount to divide falls short of what was assumed. Here again we can ignore the initial division and apply the rule to the revised problem, or we can apply the rule to the problem in which the initial claims are substituted by the (unfeasible) allocation initially proposed. The next requirement is that both ways of proceeding should result in the same awards vectors. Formally, a rule $R$ satisfies composition down if, for each $(N, c, E) \in \mathcal{D}$, and each $E^{\prime}>E$, we have $R(N, c, E)=R\left[N, R\left(N, E^{\prime}, c\right), E\right]$.

These properties are only satisfied by the constrained equal-awards rule and the constrained equal-losses rule within the reverse TAL-family. More precisely:

Proposition 6. The following statements hold:

(i) The only rules in the reverse TAL-family that satisfy composition up are $R T^{1}=L$ and $R T^{0}=A$.

(ii) The only rules in the reverse TAL-family that satisfy composition down are $R T^{1}=L$ and $R T^{0}=A$.

The same result holds for the TAL-family, i.e., the constrained equal-awards rule and the constrained equal-losses rule are also the only two rules in the TAL-family satisfying composition up (respectively, composition down), as shown by Theorem 3 in Moreno-Ternero and Villar (2006a).

There are only three symmetric rules that satisfy homogeneity, consistency, composition up and composition down. They are the proportional rule, the constrained equal-awards rule and the constrained equal-losses rule (Moulin, 2000). ${ }^{7}$ Thus, Proposition 2 implies that $R T^{1}=L$ and $R T^{0}=A$ are the only rules in the reverse TAL-family that satisfy both composition up and composition down. Proposition 6 complements this corollary showing that there is no other rule within the family satisfying only one of the properties.

\footnotetext{
${ }^{7}$ The proportional rule is the rule that selects, for each $(N, c, E) \in \mathcal{D}$, the vector $\left(\frac{E}{C} \cdot c_{i}\right)_{i \in N}$. Observe that, for a given problem $(N, c, E) \in \mathcal{D}$, the rule $R T^{\theta}$ within the reverse TAL-family, where $\theta=\frac{E}{C}$, yields an allocation $R T^{E / C}(N, c, E)$ that coincides with the allocation provided by the proportional rule to this problem. Yet, there is no $\theta$ for which $R T^{\theta}$ is the proportional rule (i.e., the proportional rule is not a member of the reverse TAL-family).
} 


\subsection{Protective properties}

We now turn our attention to a group of properties that appear in the literature referring to the application of some protective criteria for agents with "very large" or "very small" claims: sustainability, independence of residual claims, exemption, and exclusion. ${ }^{8}$ They establish restrictions on the behavior of a rule when claims are very unequal. Both exemption and sustainability require that agents with relatively small claims be fully reimbursed. In the former property, smallness is defined as having a claim below equal division of the available amount. In the latter property, a claim is considered small when substituting it for the claim of any other agent whose claim is higher, there would be enough to compensate everyone. Formally, a rule $R$ satisfies sustainability if, for each $(N, c, E) \in \mathcal{D}$, and each $i \in N$, if $\sum_{j \in N} \min \left\{c_{i}, c_{j}\right\} \leq E$ then $R_{i}(N, c, E)=c_{i}$. Similarly, a rule $R$ satisfies exemption if, for each $(N, c, E) \in \mathcal{D}, c_{i} \leq \frac{E}{n}$ implies $R_{i}(N, c, E)=c_{i}$. Although they are equivalent in the two-claimant case, sustainability implies exemption in the general case of $n$ claimants (Herrero and Villar, 2002). Conversely, if a rule satisfies exemption and consistency then it also satisfies sustainability (Yeh, 2006).

Dually, one could adopt the viewpoint that agents with larger claims are given priority so that agents with very small claims should not receive anything. We say that a claim is "residual" when the aggregate excess claim relative to the agent holding this claim exceeds the worth of the amount available. That is, $E \leq \sum_{j \in N} \max \left\{0, c_{j}-c_{i}\right\}$. Independence of residual claims requires that if an agent's claim is residual, she should get nothing. Formally, a rule $R$ satisfies independence of residual claims if, for each $(N, c, E) \in \mathcal{D}, E \leq \sum_{j \in N} \max \left\{0, c_{j}-c_{i}\right\}$ implies $R_{i}(N, c, E)=0$. Exclusion requires that if an agent's claim does not reach the average loss, she gets nothing. Formally, a rule $R$ satisfies exclusion if, for each $(N, c, E) \in \mathcal{D}, c_{i} \leq$ $\frac{C-E}{n}$ implies $R_{i}(N, c, E)=0$. It is straightforward to show that independence of residual claims and exclusion are the dual properties of sustainability and exemption, respectively. Therefore, independence of residual claims implies exclusion, whereas exclusion, in conjunction with consistency, implies independence of residual claims.

The next result shows the behavior of the reverse TAL-family with respect to these protective properties.

Proposition 7. The following statements hold:

(i) The only rule in the reverse TAL-family that satisfies sustainability is $R T^{0}=A$.

\footnotetext{
${ }^{8}$ The notions of sustainability and independence of residual claims are referred as conditional full compensation and conditional null compensation, respectively, by Thomson (2003).
} 
(ii) The only rule in the reverse TAL-family that satisfies exemption is $R T^{0}=A$.

(iii) The only rule in the reverse TAL-family that satisfies independence of residual claims is $R T^{1}=L$.

(iv) The only rule in the reverse TAL-family that satisfies exclusion is $R T^{1}=L$.

The same result holds for the TAL-family, i.e., the constrained equal-awards rule is the only rule in the TAL-family satisfying sustainability (respectively, exemption) and the constrained equal-losses is the only rule in the TAL-family satisfying independence of residual claims (respectively, exclusion), as shown by Theorem 4 in Moreno-Ternero and Villar (2006a).

\subsection{Distributional effects}

Finally, we consider the effect of changes in the parameter $\theta$ that generates the reverse TALfamily on the resulting distribution corresponding to a given problem. In order to do that, we compare the allocations generated by different rules in the family, for a given problem, by means of the classical Lorenz ordering. Given $x, y \in \mathbb{R}^{n}$ satisfying $x_{1} \leq x_{2} \leq \ldots \leq x_{n}$, $y_{1} \leq y_{2} \leq \ldots \leq y_{n}$, and $\sum_{i=1}^{n} x_{i}=\sum_{i=1}^{n} y_{i}$, we say that $x$ is greater than $y$ in the Lorenz ordering if $\sum_{i=1}^{k} x_{i} \geq \sum_{i=1}^{k} y_{i}$, for each $k=1, \ldots, n-1$, with at least one strict inequality. This criterion induces a partial ordering on allocations which reflects their relative spread. When $x$ is greater than $y$ in the Lorenz ordering, the distribution $x$ is unambiguously "more egalitarian" than the distribution $y$.

We say that a rule $R$ Lorenz dominates a rule $R^{\prime}$, which we write as $R \succsim_{L} R^{\prime}$, when for each $(N, c, E) \in \mathcal{D}, R(N, c, E)$ is greater than $R^{\prime}(N, c, E)$ in the Lorenz ordering. The following result is a corollary of a more general result proved by Thomson (2008) for the CIC family.

Proposition 8. For all $\theta_{1}, \theta_{2} \in[0,1]$ with $\theta_{1} \leq \theta_{2}, R T^{\theta_{1}} \succsim_{L} R T^{\theta_{2}}$.

Proposition 8 says that all rules within the reverse TAL-family are fully ranked in terms of the Lorenz dominance criterion. A similar result holds for the TAL-family (see Theorem 1 at Moreno-Ternero and Villar, 2006b), but with a reverse order of the parameter $\theta$ (note the difference of such a parameter in the definitions of the TAL-family and reverse TAL-family).

\section{Relationship with other families}

As mentioned above, the family studied here is related to other existing families in the literature, such as its counterpart TAL-family and the more general CIC and ICI families. The recent 
developments of the literature in bankruptcy problems present other families of rules that we consider here. We relate them to the reverse TAL-family.

Chambers and Moreno-Ternero (2015) introduce the family of generalized equal-sacrifice rules. This family comprises all the parametric rules satisfying composition down. By Proposition 6 , it follows that the constrained equal-awards rule and the constrained equal-losses rule are the only generalized equal-sacrifice rules within the reverse TAL-family. Consequently, they are also the only duals of generalized equal-sacrifice rules (i.e., parametric rules satisfying composition up) within the family. ${ }^{9}$

Flores-Szwagrzak (2015) considers the sub-family of generalized equal-sacrifice rules made of those satisfying minimal rights first. ${ }^{10}$ By the above, and Proposition 4, it follows that the intersection between such a family and the reverse TAL-family is only made of the constrained equal-losses rule. ${ }^{11}$

Thomson (2015b) and Harless (2016) are also instances of recent proposals for families of rules. In both cases, the motivation is to compromise between the proportional rule and the constrained equal-awards rule (or the constrained equal-losses rules). ${ }^{12}$ As the proportional rule is not a member of the reverse TAL-family, the compromises do not belong to the reverse TAL-family either.

From a different vantage point, there has also been a recent interest in the literature to consider more general claims problems in which claims are complemented by additional information. An instance is the concept of baselines. Hougaard et al., (2013a) introduced the so-called baselines first extension operator, which associates with each rule defined for the standard model a rule for the general model in the presence of baselines. The operator proposes to first assign agents their truncated baselines, and to allocate the resulting deficit, or surplus, using a rule for the standard problem that results after embedding baselines into claims. Specifically, a deficit is allocated according to the amounts already received by the agents, whereas a surplus is allocated according to the gap between their claims and what has already been allocated to them. As mentioned by Hougaard et al., (2013a), if each individual baseline is

\footnotetext{
${ }^{9}$ They are also the only rules within the reverse TAL-family belonging to the families characterized by Moulin (2000) and Chambers (2002).

${ }^{10} \mathrm{He}$ also considers the larger family arising from this one when dropping equal treatment of equals, as well as the corresponding dual family.

${ }^{11}$ Likewise, the intersection between the dual family and the reverse TAL-family is only made of the constrained equal-awards rule.

${ }^{12}$ See also Fragnelli et al., (2011), Giménez-Gómez and Peris (2014) and Giménez-Gómez and Osorio (2015).
} 
a fixed proportion $\theta \in(0,1)$ of the corresponding claim, then the rule induced by the constrained equal-awards rule, in such an operator, would solve the problem with baselines as the corresponding member of the reverse TAL-family would solve the original problem. ${ }^{13}$

\section{Final remarks}

We have studied in this paper a one-parameter family of bankruptcy rules, the reverse TALfamily, which generalizes the reverse Talmud rule and encompasses the constrained equal-awards rule and the constrained equal-losses rule. It is also the counterpart of the so-called TAL-family, previously introduced in the literature, which also generates the Talmud rule. We have explored the behavior of the rules in the family with respect to the standard properties in the literature. Our findings are summarized in Table 1.

We observe that all the rules within the family behave extremely well with respect to the basic and monotonicity properties. The family is closed under duality, and one only needs to consider the symmetric parameter in the domain to obtain the dual rule of a given one. The parameter describing the family can actually be interpreted as a progressivity index of the rules within the family. All these aspects are shared with its counterpart family (the TAL-family). A striking difference between both families (exemplified in Propositions 4 and 5) occurs in the behavior with respect to independence and lower bound properties. Whereas the rules within the TAL-family split in two equal halves to satisfy one of the two properties within each pair of dual properties, only the extreme elements of the reverse TAL-family satisfy them.

Our results have also allowed us to scrutinize the connections between the reverse TALfamily and some other families of bankruptcy rules that have been recently singled out in the literature.

To conclude, we note that the rules within the reverse TAL-family can be characterized by parametrizing weakenings of exemption and exclusion properties described above (e.g., van de Brink et al., 2013). ${ }^{14}$ As such parametrized properties could be consider ad hoc, and thus against the spirit of the axiomatic approach, we dismiss them from the study presented here.

\footnotetext{
${ }^{13}$ More generally, and as shown by Hujick et al., (2015), the baselines first extension operator allows to recover the whole family of CIC rules from the constrained equal-awards rule, provided baselines are generic, and not necessarily a proportion of claims.

${ }^{14}$ Similarly, the rules within the TAL-family can be characterized by parametrizing lower bounds properties (e.g., Moreno-Ternero and Villar, 2006a). More recently, Arin et al., (2015) have also characterized the reverse TAL-family resorting instead to parametrized versions of additive properties, which were not considered here.
} 


\section{Appendix. Proofs of the results}

Proof of Proposition 2. As all rules within the reverse TAL-family are parametric, we obtain, following Young (1987), that all rules within the reverse TAL-family satisfy equal treatment of equals, continuity, and consistency. ${ }^{15}$ By Lemma 3 in Chambers and Thomson (2002) they also satisfy anonymity.

As for order preservation, let $\theta \in[0,1]$ and $(N, c, E) \in \mathcal{D}$ be given. We distinguish two cases.

Case 1: $E \leq \theta C$.

In this case, $R T_{i}^{\theta}(N, c, E)=\max \left\{\theta c_{i}-\lambda, 0\right\}$, for each $i \in N$. Let $i, j \in N$ be such that $c_{i} \geq c_{j}$. It is straightforward to see that $R T_{i}^{\theta}(N, c, E) \geq R T_{j}^{\theta}(N, c, E)$. Now, suppose that $\lambda<\theta c_{j} \leq \theta c_{i}$. Then, $c_{i}-R T_{i}^{\theta}(N, c, E)=c_{i}-\left(\theta c_{i}-\lambda\right) \geq c_{j}-\left(\theta c_{j}-\lambda\right)=c_{j}-R T_{j}^{\theta}(N, c, E)$. On the other hand, if $\theta c_{i} \geq \lambda \geq \theta c_{j}$, then $c_{i}-R T_{i}^{\theta}(N, c, E)=\left(1-c_{i}\right)+\lambda \geq c_{j}=c_{j}-R T_{j}^{\theta}(N, c, E){ }^{16}$ Finally, if $\lambda>\theta c_{i}$ then $c_{i}-R T_{i}^{\theta}(N, c, E)=c_{i} \geq c_{j}=c_{j}-R T_{j}^{\theta}(N, c, E)$.

Case 2: $E \geq \theta C$.

In this case, $R T_{i}^{\theta}(N, c, E)=\theta c_{i}+\min \left\{(1-\theta) c_{i}, \mu\right\}$, for each $i \in N$. Let $i, j \in N$ be such that $c_{i} \geq c_{j}$. It is straightforward to see that $R T_{i}^{\theta}(N, c, E) \geq R T_{j}^{\theta}(N, c, E)$. Now, suppose that $\mu<(1-\theta) c_{j} \leq(1-\theta) c_{i}$. In this case, $c_{i}-R T_{i}^{\theta}(N, c, E)=(1-\theta) c_{i}-\mu \geq$ $(1-\theta) c_{j}-\mu=c_{j}-R T_{j}^{\theta}(N, c, E)$. On the other hand, if $(1-\theta) c_{i} \geq \mu \geq(1-\theta) c_{j}$ then $c_{i}-R T_{i}^{\theta}(N, c, E)=(1-\theta) c_{i}-\mu \geq 0=c_{j}-R T_{j}^{\theta}(N, c, E)$. Finally, if $\mu>(1-\theta) c_{i}$, then $c_{i}-R T_{i}^{\theta}(N, c, E)=0=c_{j}-R T_{j}^{\theta}(N, c, E)$.

Finally, we turn to scale invariance. Let $(N, c, E) \in \mathcal{D}$ be given. We distinguish three cases:

Case 1: $E<\theta C$.

As $\alpha E<\alpha \theta C$ for each $\alpha>0$, it follows that $R T_{i}^{\theta}(N, c, E)=\max \left\{\theta c_{i}-\lambda, 0\right\}$, and $R T_{i}^{\theta}(N, \alpha c, \alpha E)=\max \left\{\theta \alpha c_{i}-\lambda^{\prime}, 0\right\}$, for each $i \in N$, where $\lambda$ and $\lambda^{\prime}$ are such that $\sum_{i \in N} R T_{i}^{\theta}(N, c, E)=$ $E$ and $\sum_{i \in N} R T_{i}^{\theta}(N, \alpha c, \alpha E)=\alpha E$, respectively. Now, there exists $\bar{\lambda}>0$, such that $\lambda^{\prime}=\alpha \bar{\lambda}$. Thus, $\max \left\{\theta \alpha c_{i}-\lambda^{\prime}, 0\right\}=\alpha \max \left\{\theta c_{i}-\bar{\lambda}, 0\right\}$. Assume, without loss of generality, that $c_{n}=$ $\max _{i \in N}\left\{c_{i}\right\}$. Then, it is straightforward to see that the function $H^{\theta}:\left[0, \theta c_{n}\right] \rightarrow \mathbb{R}_{+}$such that $H^{\theta}(\lambda)=\sum_{i \in N} \max \left\{\theta c_{i}-\lambda, 0\right\}$ is piecewise linear and strictly increasing. Moreover, its image corresponds to $[0, \theta C]$. Thus, for each $E \in[0, \theta C)$ there exists a unique $\lambda_{0}$ such that

\footnotetext{
${ }^{15}$ Obviously, they also satisfy weaker versions of consistency such as those used in Ju and Moreno-Ternero (2015) or Harless (2016).

${ }^{16}$ Note that $\left(1-c_{i}\right)+\lambda \geq \theta c_{j}+(1-\theta) c_{i}$.
} 
$H^{\theta}\left(\lambda_{0}\right)=E$. This implies $\lambda=\bar{\lambda}=\lambda_{0}$, and therefore, $R T_{i}^{\theta}(N, \alpha c, \alpha E)=\alpha R T_{i}^{\theta}(N, c, E)$, for each $i \in N$.

Case 2: $E>\theta C$.

As $\alpha E>\alpha \theta C$ for each $\alpha>0$, we have $R T_{i}^{\theta}(N, c, E)=\theta c_{i}+\min \left\{(1-\theta) c_{i}, \mu\right\}$, and $R T_{i}^{\theta}(N, \alpha c, \alpha E)=\theta \alpha c_{i}+\min \left\{(1-\theta) \alpha c_{i}, \mu^{\prime}\right\}$, for each $i \in N$, where $\mu$ and $\mu^{\prime}$ are such that $\sum_{i \in N} R T_{i}^{\theta}(N, c, E)=E$ and $\sum_{i \in N} R T_{i}^{\theta}(N, \alpha c, \alpha E)=\alpha E$, respectively. Now, there exists $\bar{\mu}>0$, such that $\mu^{\prime}=\alpha \bar{\mu}$. Thus, $\alpha \theta c_{i}+\min \left\{(1-\theta) \alpha c_{i}, \mu^{\prime}\right\}=\alpha\left(\theta c_{i}+\min \left\{(1-\theta) c_{i}, \bar{\mu}\right\}\right)$. Take again $c_{n}=\max _{i \in N}\left\{c_{i}\right\}$. Then, it is straightforward to see that the function $H^{\theta}:\left[0,(1-\theta) c_{n}\right] \rightarrow$ $\mathbb{R}_{+}$such that $H^{\theta}(\mu)=\sum_{i \in N} \min \left\{(1-\theta) c_{i}, \mu\right\}$ is piecewise linear and strictly increasing. Moreover, its image corresponds to $[\theta C, C]$. Thus, for each $E \in(\theta C, C]$ there exists a unique $\mu_{0}$ such that $H^{\theta}\left(\mu_{0}\right)=E$. This implies $\mu=\bar{\mu}=\mu_{0}$, and, therefore, $R T_{i}^{\theta}(N, \alpha c, \alpha E)=\alpha R T_{i}^{\theta}(N, c, E)$, for each $i \in N$.

Case 3: $E=\theta C$.

Then $R T_{i}^{\theta}(N, c, E)=\theta c_{i}$ for each $i \in N$ and therefore, the property trivially holds.

Proof of Proposition 3. It is straightforward to show that all rules within the reverse TALfamily satisfy resource monotonicity, claims monotonicity, and population monotonicity. Thus, by Proposition 1, they all satisfy linked claim-resource monotonicity (the dual property of claims monotonicity) and linked resource-population monotonicity (the dual of population monotonicity). Finally, Chun (1989) shows that resource monotonicity and consistency together imply resource-and-population uniformity. As, by Proposition 2, all rules within the reverse TALfamily are consistent, it follows that they all satisfy resource-and-population uniformity.

Proof of Proposition 4. By duality, it suffices to show one of the statements. It is well known that $R T^{0}=A$ satisfies claims truncation invariance whereas $R T^{1}=L$ does not. Let us see that no intermediate rule within the reverse TAL-family satisfies it either. Let $\theta \in(0,1)$ and consider the two-claimant problem $B=\left(\{1,2\},\left(E, \frac{E}{\theta}\right) E\right)$. It is straightforward to check that $R T^{\theta}(B)=\left(\frac{\theta E}{2}, E-\frac{\theta E}{2}\right)$. The associated problem in which claims are truncated is $(\{1,2\},(E, E), E)$, whose solution is $\left(\frac{E}{2}, \frac{E}{2}\right)$. Therefore, $R T^{\theta}(\{1,2\}, c, E) \neq$ $R T^{\theta}(\{1,2\}, t(N, E, c), E)$

Proof of Proposition 5. By duality, it suffices to show one of the statements. It is well known that $R T^{0}=A$ satisfies average truncated lower bound on awards whereas $R T^{1}=L$ does not (e.g., Moreno-Ternero and Villar, 2004). Let us see that no intermediate rule within the 
reverse TAL-family satisfies it either. Let $\theta \in(0,1)$ and consider the two-claimant problem $B=\left(\{1,2\},\left(E, \frac{E}{\theta}\right), E\right)$. It is straightforward to check that $R T^{\theta}(B)=\left(\frac{\theta E}{2}, E-\frac{\theta E}{2}\right) \neq\left(\frac{E}{2}, \frac{E}{2}\right)$. On the other hand, assume, by contradiction, that $R T^{\theta}$ satisfies average truncated lower bound on awards, for some $\theta \in(0,1)$. Then, $R T^{\theta}(B)=\left(\frac{E}{2}, \frac{E}{2}\right)$, which represents a contradiction.

Proof of Proposition 6. (i) The rules $R T^{1}=L$ and $R T^{0}=A$ satisfy composition up (e.g., Moulin, 2000). Let us see that there is no other rule within the reverse TAL-family for which this happens. Let $\theta \in(0,1)$ be given. Consider the two-claimant problem

$$
(N, c, E)=\left(\{1,2\},\left(\frac{1}{3 \theta}, \frac{1}{\theta}\right), 1\right)
$$

and let $E_{1}=\frac{1}{2}=E_{2}$. Then, $E_{1}<E=1<\frac{1}{3}+1=\theta \cdot\left(c_{1}+c_{2}\right)$. Thus, $R T^{\theta}(N, c, E)=$ $L(N, \theta c, E)=\left(\frac{1}{6}, \frac{5}{6}\right)$ and $R T^{\theta}\left(N, c, E_{1}\right)=L\left(N, \theta c, E_{1}\right)=\left(0, \frac{1}{2}\right)$.

Let $c^{\prime}=c-R T^{\theta}\left(N, c, E_{1}\right)=\left(\frac{1}{3 \theta}, \frac{1}{\theta}-\frac{1}{2}\right)$. Then, $E_{2}-\theta \cdot\left(c_{1}^{\prime}+c_{2}^{\prime}\right)=\frac{1}{2}-\left(\frac{8-3 \theta}{6}\right)<0$, so that

$$
R T^{\theta}\left(N, c^{\prime}, E_{2}\right)=L\left(N, \theta c^{\prime}, E_{2}\right)=\left\{\begin{array}{ll}
\left(0, \frac{1}{2}\right) & \text { if } \theta \leq \frac{1}{3} \\
\left(\frac{1}{3}-\frac{5-3 \theta}{12}, \frac{2-\theta}{2}-\frac{5-3 \theta}{12}\right) & \text { if } \theta>\frac{1}{3}
\end{array} .\right.
$$

Thus, $R T^{\theta}(N, c, E) \neq R T^{\theta}\left(N, c, E_{1}\right)+R T^{\theta}\left(N, c^{\prime}, E_{2}\right)$ for each $\theta \in(0,1)$.

(ii) Both $A$ and $L$ satisfy composition down (e.g., Moulin, 2000). Now, suppose that there exists some $\theta \in(0,1)$ such that $R T^{\theta}$ satisfies composition down. Then $R T^{1-\theta}$, the dual rule of $R T^{\theta}$, should satisfy composition up, which contradicts part (i) of this theorem.

Proof of Proposition 7. Yeh (2006) shows that the constrained equal-awards rule is the only rule that satisfies sustainability and claims monotonicity. By duality, the constrained equal losses rule is the only rule that satisfies independence of residual claims and linked claimresource monotonicity. Yeh (2006) also shows that the constrained equal-awards rule is the only rule satisfying exemption, order preservation, and consistency. By duality, the constrained equal-losses rule is the only rule satisfying exclusion, order preservation and consistency. ${ }^{17}$ As all rules within the reverse TAL-family are consistent and order preserving (Proposition 2), and satisfy claims monotonicity and linked claim-resource monotonicity (Proposition 3) the proofs of all the statements follow from Yeh's results and duality.

\footnotetext{
${ }^{17}$ Note that order preservation and consistency are self-dual properties.
} 


\section{References}

[1] Arin, J., Benito-Ostolaza, J., Inarra, E., (2015) The RTAL-Family of rules for bankruptcy problems: a characterization. Mimeo. University of the Basque Country.

[2] Aumann R.J., Maschler M., (1985) Game theoretic analysis of a bankruptcy problem from the Talmud. Journal of Economic Theory 36, 195-213.

[3] Bergantiños, G., Lorenzo, L. (2008) The equal award principle in problems with constraints and claims. European Journal of Operational Research, 1881, 224-239.

[4] van den Brink, R., Funaki Y., van der Laan, G., (2013) Characterization of the reverse Talmud bankruptcy rule by exemption and exclusion properties. European Journal of Operational Research 228, 413-417.

[5] Casas-Méndez, B., Fragnelli, V., García-Jurado, I., (2011) Weighted bankruptcy rules and the museum pass problem. European Journal of Operational Research 215, 161-168.

[6] Chambers, C.P., (2005) Asymmetric rules for claims problems without homogeneity, Games and Economic Behavior 54, 241-260.

[7] Chambers, C.P., Moreno-Ternero, J.D., (2015) Taxation and poverty, Social Choice and Welfare. Forthcoming.

[8] Chambers, C.P., Thomson, W., (2002) Group order preservation and the proportional rule for bankruptcy problems, Mathematical Social Sciences 44, 235-252.

[9] Chun, Y., (1999) Equivalence of axioms for bankruptcy problems, International Journal of Game Theory 28, 511-520.

[10] Chun, Y., J. Schummer, and W. Thomson, (2001) Constrained egalitarianism: a new solution for claims problems. Seoul Journal of Economics 14, 269-297.

[11] Flores-Szwagrzak, K. (2015). Priority classes and weighted constrained equal-awards rules for the claims problem. Journal of Economic Theory 160, 36-45.

[12] Giménez-Gómez, J.M., Osorio, A., (2015) Why and how to differentiate in claims problems? An axiomatic approach. European Journal of Operational Research 241, 842-850. 
[13] Giménez-Gómez, J.M., Peris, J.E., (2014) A proportional approach to claims problems with a guaranteed minimum. European Journal of Operational Research 232, 109-116.

[14] Harless, P., (2016), Endowment additivity and the weighted proportional rules for adjudicating conflicting claims. Economic Theory. Forthcoming

[15] Herrero C, Villar A (2002) Sustainability in bankruptcy problems, TOP 10, 261-273.

[16] Hougaard J.L., Moreno-Ternero J.D., Østerdal, L.P., (2012) A unifying framework for the problem of adjudicating conflicting claims. Journal of Mathematical Economics 48, 107-114.

[17] Hougaard J.L., Moreno-Ternero J.D., Østerdal, L.P., (2013a) Rationing in the presence of baselines, Social Choice and Welfare 40, 1047-1066.

[18] Hougaard J.L., Moreno-Ternero J.D., Østerdal, L.P., (2013b) Rationing with baselines: the composition extension operator, Annals of Operations Research 211, 179-191.

[19] Huijink, S., Borm, P.E.M., Kleppe, J., Reijnierse, J.H., (2015) Bankruptcy and the per capita nucleolus: The claim-and-right rules family, Mathematical Social Sciences 77, 15-31.

[20] Ju, B-G., Moreno-Ternero, J.D., (2015). Fair allocation of disputed properties. International Economic Review. Forthcoming.

[21] Moreno-Ternero, J.D., (2007) Bankruptcy rules and coalitional manipulation. International Game Theory Review 9, 105-118.

[22] Moreno-Ternero, J.D., (2011a) Voting over piece-wise linear tax rules. Journal of Mathematical Economics 47, 29-36.

[23] Moreno-Ternero, J.D., (2011b) A coalitional procedure leading to a family of bankruptcy rules. Operations Research Letters 39, 1-3.

[24] Moreno-Ternero J.D., Villar A., (2004) The Talmud rule and the securement of agents' awards. Mathematical Social Sciences 47, 245-257.

[25] Moreno-Ternero, J.D., Villar, A., (2006a) The TAL-family of rules for bankruptcy problems. Social Choice and Welfare 27, 231-249. 
[26] Moreno-Ternero J.D., Villar A., (2006b) On the relative equitability of a family of taxation rules. Journal of Public Economic Theory 8, 283-291.

[27] Moulin H., (2000) Priority rules and other asymmetric rationing methods, Econometrica 68, 643-684.

[28] O’Neill B., (1982) A problem of rights arbitration from the Talmud, Mathematical Social Sciences 2, 345-371.

[29] Pulido M., Sanchez-Soriano J. and Llorca N., (2002), Game theory techniques for university management: an extended bankruptcy model, Annals of Operations Research 109, 129142.

[30] Pulido M., Borm P., Hendricx R., Llorca N. and Sanchez-Soriano J. (2008), Compromise solutions for bankruptcy situations with references, Annals of Operations Research 158, 133-141.

[31] Thomson, W., (2003) Axiomatic and game-theoretic analysis of bankruptcy and taxation problems: a survey, Mathematical Social Sciences 45, 249-297.

[32] Thomson, W., (2008) Two families of rules for the adjudication of conflicting claims. Social Choice and Welfare 31, 667-692.

[33] Thomson, W., (2013) The Theory of Fair Allocation, Princeton University Press, forthcoming

[34] Thomson, W., (2014) How to divide when there isn't enough: From the Talmud to game theory, Econometric Society Monograph. Forthcoming.

[35] Thomson W., (2015a) Axiomatic and game-theoretic analysis of bankruptcy and taxation problems: an update. Mathematical Social Sciences 74, 41-59

[36] Thomson, W., (2015b) For claims problems, a compromise between the constrained equal awards and proportional rules. Economic Theory 60, 495-452

[37] Timoner, P., Izquierdo, J.M., (2016) Rationing problems with ex-ante conditions. Mathematical Social Sciences 79, 46-52.

[38] Yeh, C.-H., (2006), Protective properties and the constrained equal-awards rule for claims problems: A note, Social Choice and Welfare 27, 221-230. 
[39] Young H.P., (1987) On dividing an amount according to individual claims or liabilities, Mathematics of Operations Research 12, 398-414. 
Table 1: Structural properties of the reverse TAL-family

\begin{tabular}{|c|c|}
\hline Properties & Rules that satisfy the properties \\
\hline Anonymity & $R T^{\theta}$ for each $\theta \in[0,1]$ \\
\hline Continuity & $R T^{\theta}$ for each $\theta \in[0,1]$ \\
\hline Consistency & $R T^{\theta}$ for each $\theta \in[0,1]$ \\
\hline Order preservation & $R T^{\theta}$ for each $\theta \in[0,1]$ \\
\hline Scale Invariance & $R T^{\theta}$ for each $\theta \in[0,1]$ \\
\hline Resource monotonicity & $R T^{\theta}$ for each $\theta \in[0,1]$ \\
\hline Claims monotonicity & $R T^{\theta}$ for each $\theta \in[0,1]$ \\
\hline Linked claim-resource monotonicity & $R T^{\theta}$ for each $\theta \in[0,1]$ \\
\hline Population-and-resource monotonicity & $R T^{\theta}$ for each $\theta \in[0,1]$ \\
\hline Population-and-resource uniformity & $R T^{\theta}$ for each $\theta \in[0,1]$ \\
\hline Self-duality & $R T^{1 / 2}=R T$ \\
\hline Claims truncation invariance & $R T^{0}=A$ \\
\hline Minimal rights first & $R T^{1}=L$ \\
\hline Average truncated lower bound on awards & $R T^{0}=A$ \\
\hline Average truncated lower bound on losses & $R T^{1}=L$ \\
\hline Composition up & $R T^{1}=L, R T^{0}=A$ \\
\hline Composition down & $R T^{1}=L, R T^{0}=A$ \\
\hline Exclusion & $R T^{1}=L$ \\
\hline Exemption & $R T^{0}=A$ \\
\hline Independence of residual claims & $R T^{1}=L$ \\
\hline Sustainability & $R T^{0}=A$ \\
\hline
\end{tabular}

